\title{
5G Technology: Why Should We Expect a shift from RF-Induced Brain Cancers to Skin Cancers?
}

\author{
Mehdizadeh A. R. ${ }^{1 \oplus}$, Mortazavi S. M. J., ${ }^{1,2^{*}}$
}

A report published in the New York Times "The 5G Health Hazard, That Isn't, How one scientist and his inaccurate chart led to unwarranted fears of wireless technology" addressed the controversies over the safety of 5G technology. Unfortunately, the approach of New York Times in this report is not scientific. William J. Broad in his report published July 16, 2019 criticized Dr. Bill P Curry for not considering the so called "protective effect of human skin". As a consultant and physicist, in 2000 Bill P Curry was invited to study the health risks associated with the use of laptops and wireless networks in 250,000 students in Broward County public schools in Florida. His graph labeled "Microwave Absorption in Brain Tissue (Grey Matter)" showed that electromagnetic fields are "likely to be a serious health hazard."

Broad blames Dr. Curry for not considering the low penetration of high frequencies and hence not paying attention to the shielding effect of the human skin "His analysis failed to recognize the protective effect of human skin. At higher radio frequencies, the skin acts as a barrier, shielding the internal organs, including the brain, from exposure. Human skin blocks the even higher frequencies of sunlight".

It seems that the author believes that as the outermost layer of our epidermis, the outer layer of our skin, is basically dead cells, therefore the living cells in our skin are not damaged by $5 \mathrm{G}$ radiofrequency radiation. Given this consideration, this report tries to convince the readers to accept the author's opinion that exposure to the $5 \mathrm{G}$ high frequencies is not a real concern. Moving to ionizing radiation, alpha particle can be a good analogy for this claim. However, the depth of penetration in human tissue (skin, muscle and tissues with high water content) for a frequency of $10 \mathrm{GHz}$ which lies in $5 \mathrm{G}$ frequency spectrum is about a few $\mathrm{mm}$. Given this consideration, high frequency $5 \mathrm{G}$ radiofrequency electromagnetic fields (RF-EMF) easily penetrate living skin cells and can make them severely damaged. Moreover, stating "Human skin blocks the even higher frequencies of sunlight" is misleading the people because although skin blocks the sunlight, this exposure increases the risk of skin cancer.

The non-ionizing 5G RF-EMF can behave like high LET ionizing radiations which have the maximum energy deposition per unit distance. Considering the low penetration and very high energy deposition per unit distance of $5 \mathrm{G}$, this can lead to generation of high levels of free radicals in a short distance which in turn increases the risk of skin cancer. It's worth noting that Yakymenko et al. have reported that among 100 peer-reviewed studies regarding the oxidative effects of low-intensity radiofrequency radiation that were available at the time of their study, in general, 93 confirmed that radiofrequency radiation induced oxidative effects in biological systems [1].
${ }^{1} \mathrm{PhD}$, Medical Physics Department, School of Medicine, Shiraz University of Medical Sciences, Shiraz, Iran

${ }^{2} \mathrm{PhD}$, Diagnostic Imaging Department, Fox Chase Cancer Center, Philadelphia, Pennsylvania 19111, United States

*Corresponding author: S. M. J. Mortazavi Medical Physics Department, School of Medicine, Shiraz University of Medical Sciences, Shiraz, Iran

E-mail: mortazavismj@ gmail.com

Received: 24 July 2019 Accepted: 30 July 2019 
Oxidative stress necessarily doesn't increase the chance of cancer. Although direct damage to DNA can be considered a key event, it is not sufficient alone to cause cancer because the ability of oxygen radicals and other reactive species (RS) to suppress apoptosis, and promote proliferation, invasiveness and metastasis and even angiogenesis should also be taken into account [2].

Regarding the safety of $5 \mathrm{G}$, it should be noted that like earlier technologies ( $3 \mathrm{G}$ and $4 \mathrm{G}$ ), a threshold level for detrimental effects is expected. Major shortcomings of the reports claiming no link between exposure to RF-EMF and brain cancer have been already addressed [3-6]. Furthermore, the key parameter of the large difference in the levels of RF-EMFs exposures in different studies has been introduced as the origin of some of the current controversies regarding the relationship between mobile phone usage and cancer [7]. Given this consideration, a nonlinear J-shaped dose-response relationship for the carcinogenesis of nonionizing RF-EMF is introduced. This J-shaped dose-response relationship easily explains why it is unlikely to detect brain cancers induced by exposure to RF-EMF at low levels. Moreover, a J-shaped dose-response relationship reduces the irrational fear of the risks associated with RFEMF exposures at very low levels (e.g. people who use their mobile phones occasionally). New studies support our previously proposed J-shaped dose-response relationship and show even hormetic effects at low levels while at high levels an irreversible damage and hence increased risk of cancer would be possible [8]. To investigate whether RF-EMFs are genotoxic, Sun et al. compared the effects of $1,800 \mathrm{MHz}$ RF-EMF exposure on genomic DNA in mouse embryonic fibroblasts (MEFs) with proficient (Atm $+/+$ ) or deficient (Atm-/-) ATM. They reported that their observations were similar to the hermetic effects of low doses of toxic substances "In conclusion, we report a hormesis-like effect of 1,800 MHz RF-EMF exposure on DNA damage in Atm+/+ and Atm-/- MEFs" [9]. In summary, although 5G technology brings new risks, it should be noted that regarding mobile phone use and cancer, the level of exposure is a factor that really matters.

\section{Conflict of Interest}

None

\section{References}

1. Yakymenko I, Tsybulin 0, Sidorik E, Henshel D, Kyrylenko 0, Kyrylenko S. Oxidative mechanisms of biological activity of low-intensity radiofrequency radiation. Electromagn Biol Med. 2016;35:186-202. doi: 10.3109/15368378.2015.1043557. PubMed PMID: 26151230.

2. Halliwell B. Oxidative stress and cancer: have we moved forward? Biochem J. 2007;401:1-11. doi: 10.1042/ BJ20061131. PubMed PMID: 17150040.

3. Mortazavi SAR, Mortazavi G, Mortazavi SMJ. Comments on "Radiofrequency electromagnetic fields and some cancers of unknown etiology: An ecological study". Sci Total Environ. 2017;609:1. doi: 10.1016/j.scitotenv.2017.07.131. PubMed PMID: 28732291.

4. Mortazavi SAR, Mortazavi G, Mortazavi SMJ. Use of cell phones and brain tumors: a true association? Neurol Sci. 2017;38:2059-60. doi: 10.1007/s10072-017-3055-x. PubMed PMID: 28689225.

5. Mortazavi SMJ, Mortazavi SAR, Paknahad M. Cancers of the Brain and CNS: Global Patterns and Trends in Incidence. J Biomed Phys Eng. 2018;8:151-2. PubMed PMID: 29732351; PubMed Central PMCID: PMCPMC5928307.

6. Mortazavi SMJ. Comments on analysis of mobile phone use among young patients with brain tumors in Japan. Bioelectromagnetics. 2017;38:653-4. doi: 10.1002/bem.22082. PubMed PMID: 28980326.

7. Mortazavi S, Mishra KP. Mobile phone use and cancer: Does dose really matter? Journal of Radiation and Cancer Research. 2017;8:165. doi: 10.4103/jrcr.jrcr_39_17.

8. Mortazavi S, Mortazavi S, Haghani M. Evaluation of the validity of a Nonlinear J-shaped dose-response relationship in cancers induced by exposure to radiofrequency electromagnetic fields. Journal of Biomedical Physics and Engineering. 2019;9. doi: 10.31661/jbpe.v0i0.771.

9. Sun C, Wei X, Fei Y, Su L, Zhao X, Chen G, et al. Mobile phone signal exposure triggers a hormesis-like effect in Atm(+/+) and Atm(-/-) mouse embryonic fibroblasts. Sci Rep. 2016;6:37423. doi: 10.1038/srep37423. PubMed PMID: 27857169; PubMed Central PMCID: PMCPMC5114646. 